OPEN ACCESS

Edited by:

Keng Lin Wong,

Duke-NUS Medical School, Singapore

Reviewed by:

Stefan Schob,

University Hospital in Halle, Germany

Tang Xiangyu,

Huazhong University of Science and

Technology, China

*Correspondence:

Leonard L. Yeo

leonardyeol/@gmail.com

Specialty section:

This article was submitted to

Personalized Medicine

a section of the journa

Frontiers in Digital Health

Received: 29 November 2021 Accepted: 31 December 2021

Published: 04 February 2022

Citation:

Yeo LL, Arnberg F, Chireh A,

Sharma V, Tan B, Gontu V, Little P and Holmin S (2022) The Role of Carbon

Dioxide in the Rat Acute Stroke

Penumbra.

Front. Digit. Health 3:824334. doi: 10.3389/fdgth.2021.824334

\section{The Role of Carbon Dioxide in the Rat Acute Stroke Penumbra}

\author{
Leonard L. Yeo ${ }^{1,2,3,4 *}$, Fabian Arnberg 1,2, Arvin Chireh ${ }^{2}$, Vijay Sharma ${ }^{3,4}$, Benjamin Tan $^{3,4}$, \\ Vamsi Gontu ${ }^{1,2}$, Philip Little ${ }^{1,2}$ and Staffan Holmin ${ }^{1,2}$ \\ ${ }^{1}$ Departments of Neuroradiology, Karolinska University Hospital, Stockholm, Sweden, ${ }^{2}$ Department of Clinical Neuroscience, \\ Karolinska Institutet, Stockholm, Sweden, ${ }^{3}$ Division of Neurology, Department of Medicine, National University Health \\ System, Singapore, Singapore, ${ }^{4}$ Yong Loo Lin School of Medicine, National University of Singapore, Singapore, Singapore
}

Purpose: The vasodilatory response to inhaled $\mathrm{CO}_{2}$ occurs in the acute stroke ischemic penumbra and may be a potential therapeutic modality.

Methods: Twenty-two Sprague-Dawley rats were subjected to 90-min occlusion of the M2 segment of the middle cerebral artery (M2CAO) by endovascular technique. The animals were administered different $\mathrm{C} 02$ concentrations and scanned serially with 9.4 T MRI. Infarct tissue was determined by diffusion-weighted imaging (DWI) and hypoperfused tissue was determined by arterial spin labeling (PWI).

Results: 4 animals were administered room air (RA)+6\% $\mathrm{CO}_{2}$ (group 1), 6 animals $\mathrm{RA}+12 \% \quad \mathrm{CO}_{2}$ (Group 2) and 4 animals only RA (group 3). In the rats with $\mathrm{CO}_{2}$ administered (groups 1 and 2), the DWI lesion to cerebral hypoperfusion volume ratio (SD) at pre- $\mathrm{CO}_{2}$ administration, was $0.145(0.168)$, which increased to $0.708(0.731)$ during $\mathrm{CO}_{2}$ administration and reduced to $0.533(0.527)$ post- $\mathrm{CO}_{2}$ administration. In 9 of 10 rats the hypoperfused volume decreased when $\mathrm{CO}_{2}$ was administered. When $\mathrm{CO}_{2}$ was stopped the hypoperfused volume became larger again. Administration of $\mathrm{RA}+12 \% \mathrm{CO}_{2}$ (Group 2) decreased the volume of CBF hypoperfusion significantly compared to the control group (95\% Cl: $0.084 \pm 0.0213, p=0.004)$.

Conclusion: Inhaled $\mathrm{CO}_{2}$ appears to reduce the size of the hypoperfused tissue volume during acute stroke and may be a potential modality for treatment of acute ischemic stroke. These findings will nonetheless need to be validated in a larger cohort in other centers.

Keywords: carbon dioxide, acute stroke, arterial spin label (ASL) MRI, Rat-brain, penumbra, middle cerebral arterial occlusion

\section{INTRODUCTION}

Stroke is the second most common cause of death worldwide and the major cause of disability (1). During an acute ischemic stroke, the leptomeningeal collateral circulation keeps the ischemic penumbra in the brain from succumbing to infarction (2). Studies have shown that in some patients, the ischemic penumbra can last hours or even days before it becomes infarcted (3). The discovery of factors that can affect the perfusion by modulating the leptomeningeal collaterals and sustaining the penumbra will allow more patients to be treated by recanalization therapies.

Carbon dioxide $\left(\mathrm{CO}_{2}\right)$ is one of the strongest natural vasodilators. Cerebral blood flow $(\mathrm{CBF})$ changes in concordance with changes in the partial pressure of $\mathrm{CO}_{2}\left(\mathrm{PaCO}_{2}\right)$ within the range of 
20-80 mm Hg (3). All cerebral vessels respond to alterations in $\mathrm{CO}_{2}$, but hypercapnia has been shown to dilate smaller arterioles more than larger ones $(3,4)$. Nonetheless in a hyperacute stroke, the reactivity to $\mathrm{CO}_{2}$ could be different. In swine experiments, hypocapnia was found to be associated with reduced cerebral blood flow and could increase the ischemic penumbra (5). Conversely, in humans increased $\mathrm{CO}_{2}$ has been associated with the "reversed Robin Hood syndrome" which is a steal phenomenon linked with worse clinical outcomes (6). In humans with sleep apnoea syndrome, non-invasive positive pressure ventilation to increase ventilation of $\mathrm{CO}_{2}$ has been used as an adjunctive treatment during acute stroke (7).

The reactivity of blood vessels to $\mathrm{CO}_{2}$ in stroke is well described (8-13) and there are several studies which have looked at the effects of high levels of $\mathrm{CO}_{2}$ on rodents with middle cerebral artery ligation occlusion (MCAO) which has shown a protective clinical effect $(14,15)$. However the use of $\mathrm{CO}_{2}$ as an adjunctive treatment has not gained widespread use (7). This is partially due to the worry of increased $\mathrm{CO}_{2}$ acidosis causing worse outcomes in the context of acute ischemic stroke, as well as poor understanding of the underlying physiological changes in cerebral perfusion associated with $\mathrm{CO}_{2}$ which can translate into clinical outcomes $(16,17)$.

We seek to bridge this gap and we hypothesize that $\mathrm{CO}_{2}$ will affect the leptomeningeal collateral circulation and can be used to modulate cerebral perfusion in acute ischemic stroke. Administering $\mathrm{CO}_{2}$ could either help in maintaining a penumbral tissue state or it may worsen the ischemia by stealing blood away from affected area. We therefore performed an experiment where $\mathrm{CO}_{2}$ was administered in different concentrations in rats who had acute stroke to determine if it would change the volume of the ischemic penumbra.

\section{METHODS}

All animal handling and experiments were conducted according to the guidelines provided by the Animal Welfare Board at Karolinska Institute, Stockholm, Sweden. This study was approved by the Stockholm Northern Regional Ethical Committee (Ethical approval \#N4/15). Animals in research is regulated by a common law EU framework (Directive 2010/63/ EU) and the experiments were conducted in compliance with the Animal Research: Reporting In-Vivo Experiments (ARRIVE) guidelines.

Twenty-two Male Sprague-Dawley rats (350-400 grams, approximately 14 weeks age, Scanbur B\&K, Sollentuna, Sweden) were maintained on 12:12 light:dark cycle (lights on at $0900 \mathrm{~h}$ ) and provided food and water ad libitum. Approximately during anesthesia, animals were kept normothermic by means of a rectal thermistor coupled with a heating pad.

Anesthesia was initially induced using $4 \%$ isoflurane mixed with $100 \% \mathrm{O} 2$ and subsequently maintained at $2 \%$ isoflurane concentration in an air:oxygen mixture (7:3), which was later switched to a sub-cutaneous medetomidine infusion (500 ug over $1 \mathrm{~h}$ ) before occluding the distal middle cerebral artery. The switch to medetomidine was performed to prevent vasodilatory effects of isoflurane from affecting the $\mathrm{CO}_{2}$ dilatory effects. Anesthesia was confirmed by a reduction in the breathing rate and the absence of a withdrawal reflex in response to tail pinch. Animal vital signs were monitored throughout the surgical procedure and a piezo electric force transducer mounted on the animal's thorax to determine the respiratory rate and amplitude of respiration while in the MRI scanner.

All animals were subjected to occlusion of the distal middle cerebral artery as described previously, (M2CAO) (18). Briefly, an incision was made in the tail artery and a 1.2F Magic hydrophilic microcatheter (BALT, Irvine, CA) was advanced to the proximal descending aorta carrying a 0.007 inch Hybrid microwire (Balt Extrusion, Montmorency, France). Subsequently, the tip of the microwire was navigated to, and positioned in the base of the internal carotid artery to allow blood flow past. The rats were then switched to the aforementioned sub-cutaneous medetomidine (Domitor ${ }^{\circledR}$, Pfizer) infusion and the isoflurane was allowed to wash out for approximately $30 \mathrm{~min}$, during which time the animals were placed into a restrainer consisting of a multiconcentric, acrylic head and body holder with built-in dual coil radiofrequency electronics fitted with a ventilation tube for gas inhalation. This helped to maintain spatial consistency between examinations and to reduce motion artifacts. The guidewire was then advanced to the distal middle cerebral artery causing an M2 occlusion (M2CAO). Next, the animal was transferred to a 9.4-T MRI scanner, where a continuous flow of heated air was used to maintain animal body temperature during the examination.

\section{STUDY DESIGN}

The animals were divided into three groups, those subjected to either pre-mixed air with $6 \% \mathrm{CO}_{2}$ (group 1), air with $12 \% \mathrm{CO}_{2}$ (Group 2) or room air (without supplemental $\mathrm{CO}_{2}$ ), (Group 3 ). The animals were not randomized for this study. The mole fraction of $\mathrm{O} 2$ in these gas mixtures was maintained at $21 \%$ by reducing the fraction of nitrogen. After the M2CAO was induced as described, the rats underwent MRI scanning. After the 1st imaging sequence, the pre-mixed $\mathrm{CO}_{2}$ gas was turned on and the rats underwent a second series of MRI scans. The pre-mixed $\mathrm{CO}_{2}$ gas was then turned off and the rats underwent a final series of MRI scans. At the end of the experiment, the rats were euthanized through decapitation while in deep anesthesia and both hemispheres of the brain were harvested (Figure 1).

\section{MAGNETIC RESONANCE IMAGING}

The animals were transferred to the MRI scanner within 5 min of the placement of the microwire to occlude the M2 branch of the MCA. Diffusion-weighted imaging and arterial spin labeling was obtained with three-dimensional volumetric data of the entire brain. This was performed with a MRI comprised of a horizontal 9.4T magnet (Varian, Yarnton, United Kingdom), with a $120 \mathrm{~mm}$ inner diameter gradient system and a maximum gradient strength of $600 \mathrm{mT} / \mathrm{m}$. The T2 weighted sequences were acquired with $1 \mathrm{~mm}$ thick slices in the coronal plane 


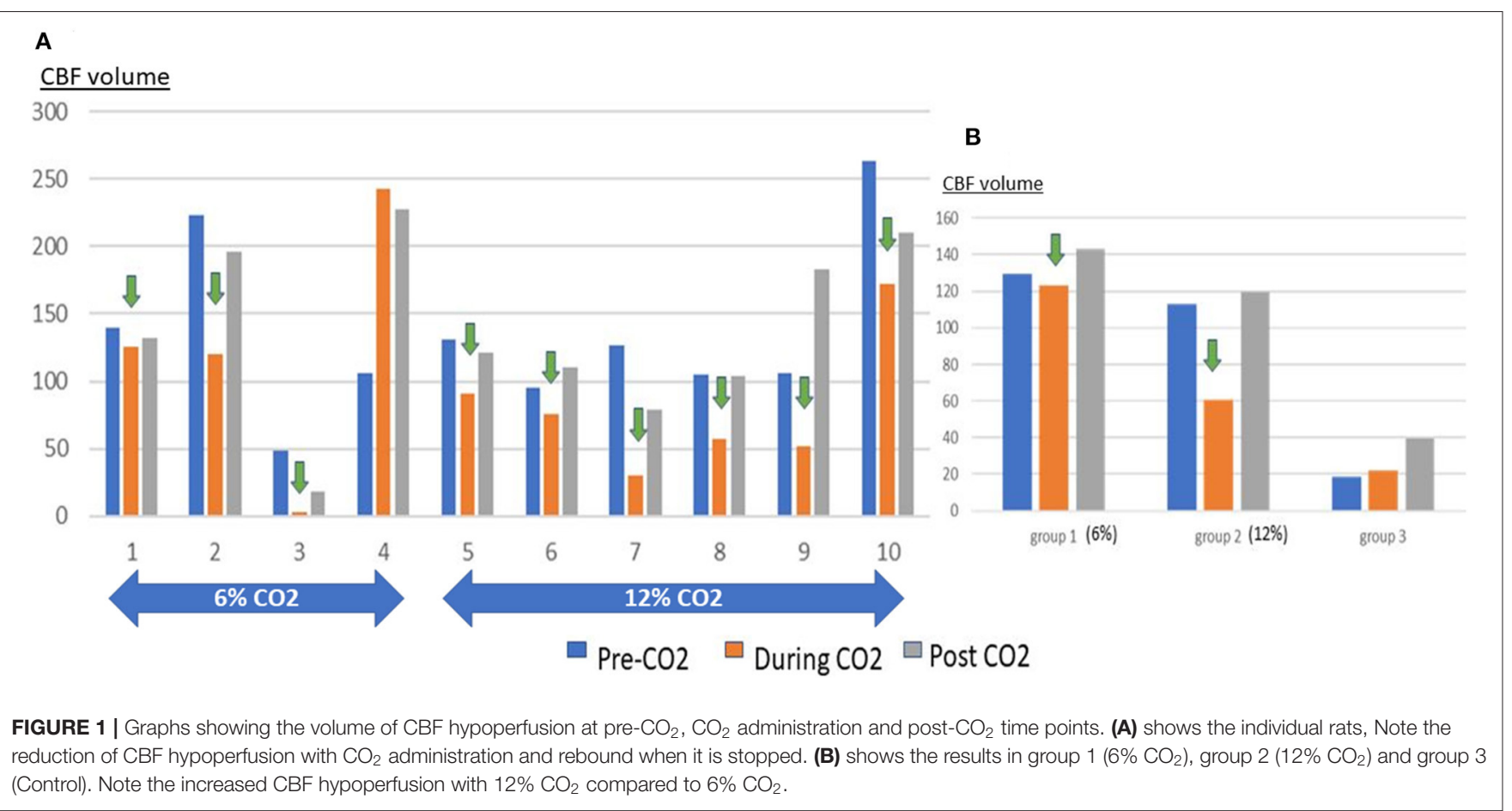

(13 slices), using multi-slice three-shot spin-echo echo-planar imaging. Using a field of view (FOV) of $32 \times 32 \mathrm{~mm}$, repetition time (TR) of $3 \mathrm{~s}$, echo time (TE) of $25 \mathrm{~ms}$ and matrix size of $96 \times 96$ zero filled to $128 \times 128$, resulted in an in-plane resolution of $256 \mu \mathrm{m}(18,19)$. Diffusion weighted images were acquired with a diffusion gradient duration $(\Delta): 2.3 \mathrm{~ms}$, diffusion gradient separation $(\delta)$ : $6.5 \mathrm{~ms}$. Diffusion sensitizing gradients were applied along 12 directions with a $b$ value $=1,000 \mathrm{~s} / \mathrm{mm}^{2}$ and a control image collected twice with the diffusion sensitizing factor $b=0$. Apparent diffusion coefficient (ADC) maps $\left(\mathrm{mm}^{2} / \mathrm{s}\right)$ were then obtained from this data $(18,19)$.

For perfusion sequences, an actively tuned arterial spin labeling (ASL) surface coil (Rapid Biomedical $\mathrm{GmbH}$, WuẼrzburg-Rimpar, Germany) was positioned under the neck of the animal. In order to have one coil tuned to the Larmor frequency at a time, the coils were actively decoupled through a pin diode driver with three independent channels (Rapid Biomedical GmbH, Wurzburg-Rimpar, Germany). The localized excitation of the neck coil did not extend to the brain, and magnetization transfer could safely be ignored with the actively decoupled three-coil configuration. PWI was acquired with continuous ASL and by single-shot gradient echo planar imaging by applying an off-resonance radio frequency power to the ASL coil simultaneously with a 1 Gauss/cm gradient during TR. The specifications for TR were: $6 \mathrm{~s}$, TE: $10.2 \mathrm{~ms}$, with the FOV and resolution were similar to the T2 weighted sequence. Tagged and non-tagged control images was acquired for each slice and there were 25 repetitions per slice. The labeling plane was located $24 \mathrm{~mm}$ up from the center of the applied slice package. This corresponds to $7 \mathrm{kHz}$ off-resonance for the slice nearest to the labeling plane and increasing by $0.4 \mathrm{kHz}$ for each slice. The RF power of the tag coil is put at zero for the control image $(18,19)$. For the perfusion images, the configuration and position of the slice package was the same as the DWI and T2 weighted sequence. From this data, the cerebral blood flow (CBF) maps ( $\mathrm{ml} / \mathrm{g} / \mathrm{min}$ ) were calculated. VnmrJ (Agilent Technologies, Palo Alto, CA, USA), ImageJ (National Institutes of Health, Maryland, USA), and OsiriX (OsiriX Foundation, Geneva, Switzerland) software were used for MRI data processing $(18,19)$. Each MRI scan took $20 \mathrm{~min}$ with the optimized protocol and to keep the duration uniform between experiments, the animal was not moved between each of the 3 scans per animal (without $\mathrm{CO}_{2}$, with $\mathrm{CO}_{2}$ administered and with $\mathrm{CO}_{2}$ stopped) (Figure 1).

Regions of interest (ROI) segmentation was done with ImageJ and ITK-SNAP software, during which the assessor was blinded to which group the animals were from. Each image was visually inspected, and individual ROIs were manually traced areas with decreased ADC to delineate the ischemic core. ROIs were similarly manually traced around areas with reduced CBF seen on the ASL maps (Figure 2).

\section{STATISTICS}

We present the numerical variables as mean and standard deviation (SD). Analysis of changes in volumes of cerebral perfusion at different $\mathrm{CO}_{2}$ concentrations were tested by using 2-sample $t$-test or Mann-Whitney $U$ test where applicable. Associations were presented as odds ratios (OR) with corresponding 95\% confidence intervals (CI). Statistical significance was defined as $p<0.05$. Statistical analyses were performed using the Statistical Package for Social Sciences (SPSS) version 21. 


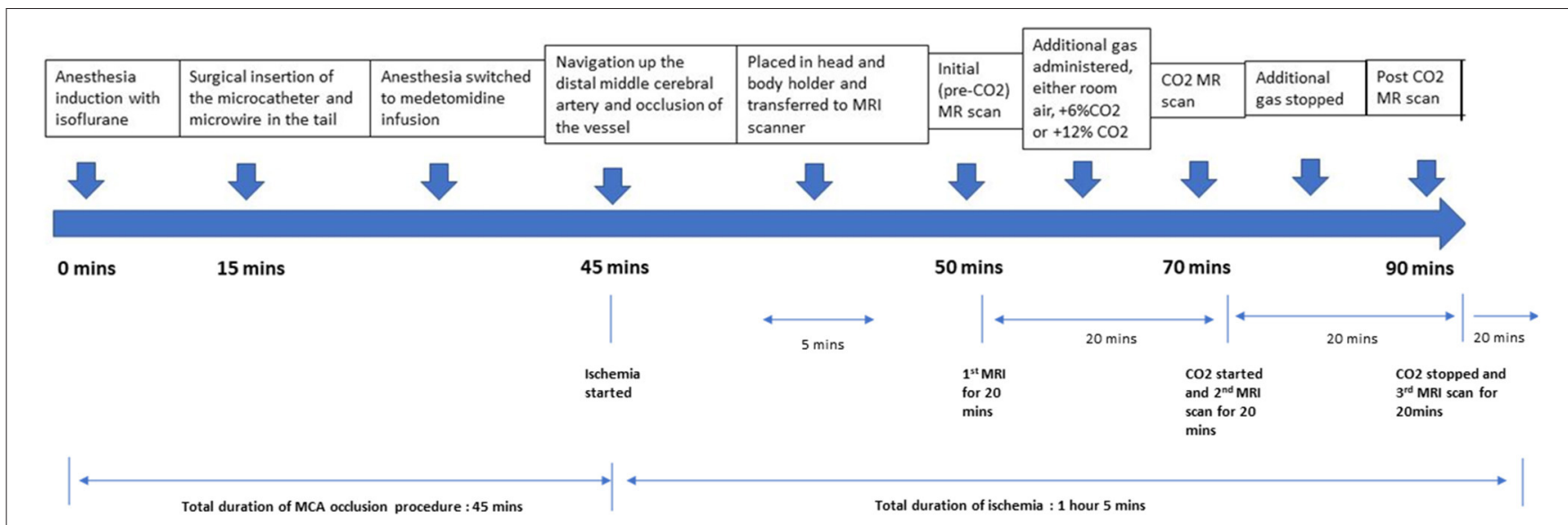

FIGURE 2 | Timeline of the experiment, with approximate timing of each stage of the experiment below.

TABLE 1 | Ipsilateral ratio of diffusion weighted imaging (DWI) volume and arterial spin labeling hypoperfusion (CBHF) volume divided by ipsilateral hemispheric brain volume, at various time points and in the three groups.

\begin{tabular}{|c|c|c|c|c|c|c|c|c|c|c|}
\hline $\mathbf{S} / \mathbf{N}$ & $\begin{array}{l}\text { Additional } \\
\mathrm{CO}_{2} \\
\text { concentration }\end{array}$ & $\begin{array}{c}\text { Pre-CO } \\
\text { DWI }\end{array}$ & $\mathbf{C O}_{2}$ DWI/ & $\begin{array}{c}\text { Post-CO } \\
\text { DWI }\end{array}$ & $\begin{array}{c}\text { Pre-CO } \\
\text { CBFH }\end{array}$ & $\mathrm{CO}_{2} \mathbf{C B F H}$ & $\begin{array}{c}\text { Post-CO } \\
\text { CBFH }\end{array}$ & $\begin{array}{c}\text { pre-CO } \\
\text { DWI/CBFH }\end{array}$ & $\begin{array}{c}\mathrm{CO}_{2} \\
\mathrm{DWI} / \mathrm{CBFH}\end{array}$ & $\begin{array}{c}\text { Post-CO } \\
\text { DWI/CBFH }\end{array}$ \\
\hline Rat 1 & $6 \%$ & 0.005 & Not done & Not done & 0.198 & 0.179 & 0.187 & 0.026 & Not done & Not done \\
\hline Rat 2 & $6 \%$ & 0.082 & 0.086 & 0.092 & 0.319 & 0.172 & 0.281 & 0.258 & 0.497 & 0.327 \\
\hline Rat 3 & $6 \%$ & 0.007 & 0.003 & 0.006 & 0.062 & 0.004 & 0.023 & 0.107 & 0.795 & 0.256 \\
\hline Rat 4 & $6 \%$ & 0.081 & 0.144 & 0.139 & 0.143 & 0.329 & 0.307 & 0.568 & 0.437 & 0.453 \\
\hline Rat 5 & $12 \%$ & 0.024 & 0.009 & 0.027 & 0.172 & 0.118 & 0.158 & 0.142 & 0.077 & 0.168 \\
\hline Rat 6 & $12 \%$ & 0.005 & 0.004 & 0.013 & 0.127 & 0.101 & 0.148 & 0.042 & 0.037 & 0.090 \\
\hline Rat 7 & $12 \%$ & 0.002 & 0.031 & 0.061 & 0.173 & 0.042 & 0.109 & 0.011 & 0.736 & 0.558 \\
\hline Rat 8 & $12 \%$ & 0.014 & 0.201 & 0.276 & 0.151 & 0.081 & 0.149 & 0.095 & 2.467 & 1.848 \\
\hline Rat 9 & $12 \%$ & 0.004 & 0.066 & 0.098 & 0.139 & 0.067 & 0.240 & 0.028 & 0.979 & 0.410 \\
\hline Rat 10 & $12 \%$ & 0.063 & 0.083 & 0.199 & 0.360 & 0.236 & 0.288 & 0.175 & 0.350 & 0.689 \\
\hline Control 1 & Nil & 0.123 & 0.098 & 0.104 & 0.074 & 0.075 & 0.126 & 1.655 & 1.310 & 0.821 \\
\hline Control 2 & Nil & 0.005 & Not done & Not done & 0.002 & 0.016 & Not done & 2.125 & Not done & Not done \\
\hline Control 3 & Nil & 0.023 & 0.008 & 0.010 & 0.007 & 0.018 & 0.027 & 3.368 & 0.443 & 0.377 \\
\hline Control 4 & Nil & 0.002 & 0.002 & Not done & 0.014 & 0.006 & Not done & 0.163 & 0.418 & Not done \\
\hline
\end{tabular}

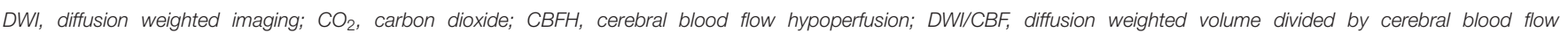
hypoperfusion volume.

\section{RESULTS}

An initial total of 22 animals had the M2CAO stroke procedure performed. 9 rats with $6 \% \mathrm{CO}_{2}$ administered (group 1), 9 rats with $12 \% \mathrm{CO}_{2}$ (Group 2) and 4 control rats without additional $\mathrm{CO}_{2}$ (group 3). Three animals in Group 1 and one animal in group 2 were excluded due to subarachnoid hemorrhage secondary to perforation of the MCA. One animal from group 2 was excluded due to absence of infarct on MRI. Two animals from group 1 and 1 animal from Group 2 died before transfer to the MRI scanner. In Rat 1 (from group 1) and control 2 (from group 3), the MRI scanner technical malfunction resulted in data loss of the DWI images during $\mathrm{CO}_{2}$ administration and post$\mathrm{CO}_{2}$ administration. The post- $\mathrm{CO}_{2}$ administration DWI and
ASL images were also lost for control 2 and control 4. Finally, Data were tabulated for each of the groups; room air $+6 \%$ $\mathrm{CO}_{2}$ (group1, $n=4$ ), inhaling room air $+12 \% \mathrm{CO}_{2}$ (group 2, $n=6$ ) and inhaling room air with no additional $\mathrm{CO}_{2}$ (control group, $n=4)$. All images were viewed and assessed for motion artifacts (Table 1).

In rats administered $\mathrm{CO}_{2}$ (Group 1 and 2 combined), the mean volume of infarcted tissue as measured by the ratio of the ADC lesion to the hemispheric volume ( $\mathrm{mm} 3 \pm \mathrm{SD})$ was 0.028 (0.033), $0.069(0.068)$ and $0.101(0.09)$ for pre- $\mathrm{CO}_{2}$, during $\mathrm{CO}_{2}$ and post- $\mathrm{CO}_{2}$ administration, respectively. While in the control group the ADC lesions were 0.039 (0.057), 0.036 (0.536) and $0.057(0.066)$ for the corresponding sequences. The mean volume of hypoperfused tissue as measured by ASL $\left(\mathrm{mm}^{3} \pm \mathrm{SD}\right)$ 


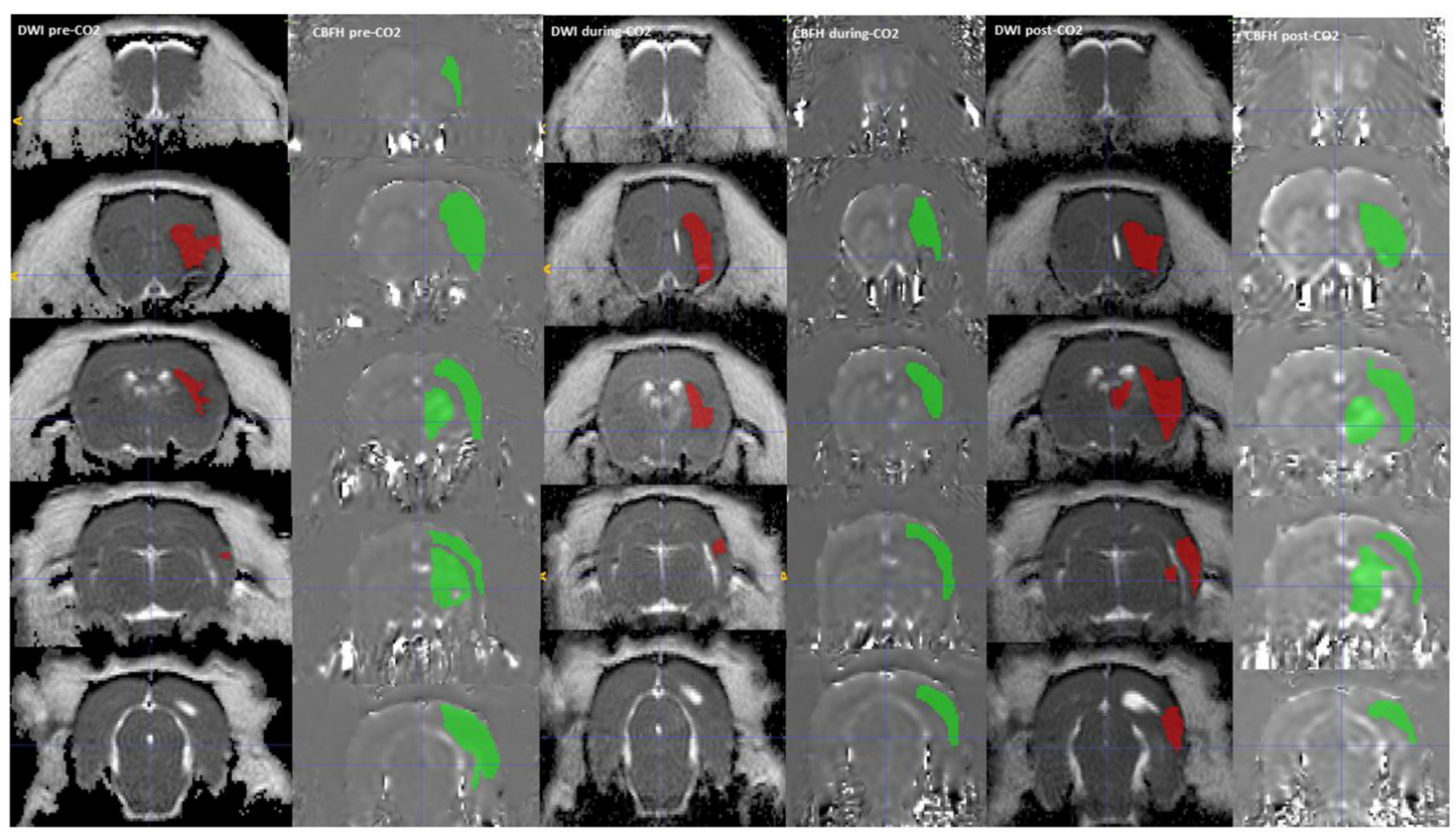

FIGURE 3 | A DWI (Green) and CBF hypoperfusion, CBFH (Red) region of interest drawn onto the corresponding MRI sequences, showing a reduction in CBF hypoperfusion volume with administration of $\mathrm{CO}_{2}$. The $\mathrm{CBF}$ hypoperfusion volume reverses and increases with stoppage of $\mathrm{CO}_{2}$.

TABLE 2 | Changes in cerebral blood flow hypoperfusion (CBFH)/hemisphere volume: 6 and $12 \%$ carbon dioxide $\left(\mathrm{CO}_{2}\right)$ groups versus control groups.

\begin{tabular}{|c|c|c|c|c|c|}
\hline & $\begin{array}{l}\text { Control group } \\
\text { Mean (SD) }\end{array}$ & $\begin{array}{c}6 \% \mathrm{CO}_{2} \text { group } \\
\text { Mean (SD) }\end{array}$ & 95\% Cl, P-value & $12 \% \mathrm{CO}_{2}$ groupMean (SD) & $95 \%, P$-value \\
\hline Pre $\mathrm{CO}_{2}$ vs. during $\mathrm{CO}_{2}$ & $-0.004(0.009)$ & $0.079(0.412)$ & -1.59 to $0.186, P=0.853$ & $0.0094(0.141)$ & 0.034 to $0.133, P=0.004$ \\
\hline During $\mathrm{CO}_{2}$ vs. post $\mathrm{CO}_{2}$ & $0.03(0.029)$ & $0.074(0.049)$ & -0.491 to $0.137, P=0.291$ & $0.283(0.056)$ & -0.124 to $0.120, P=0.966$ \\
\hline
\end{tabular}

for the whole cohort of rats administered $\mathrm{CO}_{2}$ (group 1 and 2 combined) was 0.184 (0.089), $0.133(0.098)$ and $0.189(0.090)$ for pre- $\mathrm{CO}_{2}$, during $\mathrm{CO}_{2}$ and post- $\mathrm{CO}_{2}$ administration, respectively. While in the control group the mean volume of hypoperfused tissue was $0.024(0.034), 0.029(0.313)$ and $0.767(0.069)$ for the corresponding sequences (Table 1 ).

In the rats administered $\mathrm{CO}_{2}$ (Groups 1 and 2 combined) the DWI lesion to cerebral hypoperfusion volume ratio (SD) was $0.145(0.168)$ at pre- $\mathrm{CO}_{2}$ administration, this increased to 0.708 (0.731) during $\mathrm{CO}_{2}$ administration and subsequently reduced to $0.533(0.527)$ post $-\mathrm{CO}_{2}$ administration. In 9 out of 10 rats the volume of the hypoperfused tissue decreased when $\mathrm{CO}_{2}$ was administered but this decrease reversed when the $\mathrm{CO}_{2}$ was discontinued (Figure 3). The mean decrease in $\mathrm{CBF}$ hypoperfusion was 0.051 (0.094) for all rats with $\mathrm{CO}_{2}, 0.079(0.412)$ for rats breathing room air $+12 \%$ $\mathrm{CO}_{2}$ and $0.0094(0.141)$ for rats breathing room air + $6 \% \mathrm{CO}_{2}$. Only administration of room air $+12 \% \mathrm{CO}_{2}$ decreased the volume of $\mathrm{CBF}$ hypoperfusion significantly when compared to the control group (95\%CI: 0.034-0.133, $p=0.004$ ) (Table 2). Rat 4 from group 1 did not have a reduction in $\mathrm{CBF}$ hypoperfusion with administration of $\mathrm{CO}_{2}$, this is likely due to the large infarct size which involved the hypothalamic region, which caused hyperthermia throughout the experiment (Supplementary Figure 1).

\section{DISCUSSION}

Our study shows that $\mathrm{CO}_{2}$ can reduce the volume of $\mathrm{CBF}$ hypoperfusion during acute ischemic stroke in rats and was seen in $90 \%$ of our experimental cohort. There was also a strong suggestion of a dose dependent response where the $12 \%$ $\mathrm{CO}_{2}$ group showed a significant decrease in CBF hypoperfusion compared to controls, but not the $6 \% \mathrm{CO}_{2}$ group.

Stroke is the fifth cause of death in the USA and the leading medical cause of acquired adult disability worldwide $(20,21)$. The ischemic penumbra, the area of ischemic brain tissue surrounding the infarcted core, is potentially salvageable if an appropriate treatment is administered within a specified therapeutic window. (22) There are two major approaches to treat acute ischemic stroke: neuroprotection and reperfusion. Reperfusion by thrombolysis and/or interventional thrombectomy has changed the treatment paradigm owing to their high efficacy, however, only a limited number of 
patients benefit from reperfusion therapies due mainly to time constraints. Recent efforts have been undertaken to identify acute therapies which can potentially prolong the deterioration of the ischemic penumbra, and to better understand the mechanisms by which this tissue is irreversibly damaged. However an ideal neuroprotective agent for acute ischemic stroke remains elusive (22-25).

$\mathrm{CO}_{2}$ is a strong vasodilator in the brain and can affect the cerebral blood flow with commensurate changes of up to $4 \%$ per change in $\mathrm{PaCO}_{2}$ in humans (26). In the presence of carbonic anhydrase, elevated levels of $\mathrm{CO}_{2}$ form carbonic acid in the blood, creating an acidic environment that enhances the vasodilatory effects of adenosine and increases potassium ion conductance across vascular smooth muscles. Cerebral arterial smooth muscles are sensitive to the partial pressure of $\mathrm{CO}_{2}$ in the blood, and this response appears to be mainly modulated by extracellular fluid $\mathrm{pH}$. The result is a dilation of blood vessels with decreased resistance and increased blood flow (27-29). Although all cerebral vessels appear to respond to changes in $\mathrm{PaCO}_{2}$, the vasodilatory effects appears to be more in smaller arterioles than larger ones (26).

This is the first animal acute stroke study that shows the effects of $\mathrm{CO}_{2}$ in reducing the volume of hypoperfused tissue using serial perfusion imaging, that we are aware of. In this study, the DWI volume steadily increased in the rats administered $\mathrm{CO}_{2}$, which is what is normally expected in the evolution of stroke, but the DWI/hypoperfused volume ratio fluctuated due to a reduction in the hypoperfused volume when $\mathrm{CO}_{2}$ was administered. Importantly, when the $\mathrm{CO}_{2}$ administration was stopped, there was a rebound in the volume of hypoperfusion volume showing that the reduction was indeed dependent on the $\mathrm{CO}_{2}$ and not on other factors. This could potentially mean that the ischemic penumbra in acute stroke patients could be kept viable with $\mathrm{CO}_{2}$ administration until they received recanalization therapy. We also observed a strong indication of a dose dependent response to the $\mathrm{CO}_{2}$ concentration. While there are few studies on $\mathrm{CO}_{2}$ administration in ischemic stroke, a study reported a reduction in $\mathrm{CBF}$ in rats when $\mathrm{CO}_{2}$ concentration was decreased, and this was postulated to potentially worsen the area of infarction (30). This study corroborates our findings.

Only Rat 4 in our study did not have a reduction in the CBF hypoperfusion volume with $12 \% \mathrm{CO}_{2}$ administration. Rat 4 had a large hemispherical infarct involving the hypothalamus, resulting in the high temperature ranging from 38.9 to $41^{\circ}$ that the animal sustained throughout the experiment and consequently, affected the vasodilation. Although this is a single animal and may not be generalizable, nonetheless it suggests that certain types of stroke may not respond to $\mathrm{CO}_{2}$ administration. Interestingly, rat 10 also had a similar sized stroke but the hypothalamic area was spared, and the rat showed good response to $12 \% \mathrm{CO}_{2}$ with a reduction in CBF hypoperfusion which reversed when the $\mathrm{CO}_{2}$ was stopped.

Previous experiments involving various treatment modalities for preserving the ischemic penumbra in rats have not been very effective in translating to humans. This may be due to the difference in cerebral physiology between humans and rats. The most widely used model, for focal cerebral ischemia, is the intraarterial suture occlusion of the middle cerebral artery (MCAO) in rats $(31,32)$ where a monofilament is inserted into a transected external carotid artery and extended to occlude the origin of the middle cerebral artery. The main issue with the MCAO model is the limitation of blood flow to the anterior and posterior cerebral artery resulting in extensive ischemic infarcts mimicking a large hemispheric stroke. The hemispheric occlusion inhibits collateral flow from adjacent vessels, rendering it a suboptimal model for studying the effects of the collateral circulation in stroke $(33,34)$. Secondly, in rats, the posterior cerebral artery, arising from the internal carotid terminus, is the main tributary to the thalamus, hypothalamus, hippocampus, and substantia nigra (35-37). Infarction in the above important regions lead to a variable level of increased body temperature, disturbed water homeostasis, and severe paresis, which introduces bias in experimental studies. Human strokes tend to encompass 4.5$14 \%$ of the volume of the ipsilateral hemisphere compared with $21-45 \%$ in the MCAO rat models, and therefore in combination with the limited pial collateral circulation with the MCAO model, results obtained through this model tend to not translate to human infarcts $(33,34)$.

In the present study, we used a recently described model for inducing a small focal cortical infarction that preserves collateral flow (19). Selective M2 occlusion does not disrupt collateral blood flow to the MCA from the anterior and the posterior cerebral arteries, thus producing ischemic stroke which are more similar in both size and regional blood flow to those commonly found in human patients. In a previous study by our group using the same distal MCA technique, we noted an initial rapid occurrence of both cytotoxic and vasogenic edema within the ischemic core. Although this core rapidly undergoes infarction, the subsequent spread of infarction in the initial hypoperfused area occurs at a slower pace (19), similar to a human clinically in an ischemic stroke, and this was likely due to the preserved collateral blood flow of the M2CAO model. The preservation of collateral supply and gradual onset of ischemic changes in the M2CAO stroke model are important in our study, particularly as we look for cortical CBF changes due to $\mathrm{CO}_{2}$ administration.

We acknowledge some limitations of the current study. Being an animal experiment with a small sample size has its inherent limitations and there was no randomization of the groups. Moreover, out of 22 rats, only 14 were usable for the analysis that may have introduced some bias. The other main limitation is the lack of vascular resistance measurements and endothelial or smooth muscle histology that could help elucidate the mechanism producing the differences, and the lack of validation of the infarct volume by histology. The stroke model is designed to create smaller distal M2 infarcts, however subcortical structures were sometimes be involved in the infarct. This is a problem inherent in the model, nonetheless, the volume of infarct is smaller than that seen in an MCAO model. We also recognize that $\mathrm{CBF}$ hypoperfusion is a dynamic variable which may not directly reflect the evolution of ischemia in a linear fashion and this may be affected by the collateral circulation in the animals. There was a larger CBF hypoperfusion volume and somewhat more rapid lesion growth in animals administered with $\mathrm{CO}_{2}$, compared to the control group. This may be due to combination of smaller stroke sizes induced in the control group 
and the limited sample size of the control group. The control group may also have had better collateral circulation. Finally, we are not suggesting imminent use of $\mathrm{CO}_{2}$ as an acute treatment, as further validation studies are warranted, followed by appropriate human stroke trials studies.

\section{CONCLUSION}

Carbon dioxide in acute stroke appears to improve the cerebral blood flow and reduce the size of the perfusion defect. This is a cheap and potentially useful therapeutic modality with widespread availability which could be used to help stabilize the collaterals and maintain the penumbra until recanalization therapy is performed.

\section{DATA AVAILABILITY STATEMENT}

The original contributions presented in the study are included in the article/Supplementary Material, further inquiries can be directed to the corresponding author/s.

\section{ETHICS STATEMENT}

This study was approved by the Stockholm Northern Regional Ethical Committee (Ethical approval \#N4/15).

\section{REFERENCES}

1. Donnan GA, Fisher M, Macleod M, Davis SM. Stroke. Lancet. (2008) 371:1612-23. doi: 10.1016/S0140-6736(08)60694-7

2. Jung S, Wiest R, Gralla J, McKinley R, Mattle H, Liebeskind D. Relevance of the cerebral collateral circulation in ischaemic stroke: time is brain, but collaterals set the pace. Swiss Med Wkly. (2017) 147:w14538. doi: 10.4414/ smw.2017.14538

3. Raper AJ, Kontos HA, Patterson JL Jr Response of pial precapillary vessels to changes in arterial carbon dioxide tension. Circ Res. (1971) 28:51823. doi: 10.1161/01.RES.28.5.518

4. Liebeskind DS, Kim D, Starkman S, Changizi K, Ohanian AG, Jahan $\mathrm{R}$, et al. Collateral failure? Late mechanical thrombectomy after failed intravenous thrombolysis. J Neuroimag. (2008) 20:78-82. doi: 10.1111/j.15526569.2008.00295.x

5. Albuquerque $\mathrm{ML}$, Leffler $\mathrm{CW}$. $\mathrm{pHo}, \mathrm{pHi}$ and $\mathrm{pCO} 2$ in stimulation of IP3 and $[\mathrm{Ca} 2+]$ in piglet cerebrovascular smooth muscle. Proc Soc Exp Biol. (1998) 219:226-34, doi: 10.3181/00379727219-44336

6. Alexandrov AV, Sharma VK, Lao AY, Tsivgoulis G. et al. Reversed Robin Hood syndrome in acute ischemic stroke patients. Stroke. (2007) 38:30458. doi: 10.1161/STROKEAHA.107.482810

7. Tsivgoulis G, Alexandrov AV, Katsanos AH, Barlinn K, Mikulik R. et a. Noninvasive ventilatory correction in patients with acute ischemic stroke: a systematic review and meta-analysis. Stroke. (2017) 48:22858. doi: 10.1161/STROKEAHA.117.017661

8. Dirnagl U, Pulsinelli W. Autoregulation of cerebral blood flow in experimental focal brain ischemia. J Cereb Blood Flow Metab. (1990) 10:32736. doi: $10.1038 /$ jcbfm.1990.61

9. Dettmers C, Young A, Rommel T, Hartmann A, Weingart O, Baron JC. $\mathrm{CO} 2$ reactivity in the ischemic core, penumbra and normal tissue 6 hours after acute MCA-occlusion in primates. Acta Neurochir. (1993) 125:1505. doi: 10.1007/BF01401843

\section{AUTHOR CONTRIBUTIONS}

LY designed the experiment and carried it out, analyzed and interpreted the data and wrote the manuscript. FA and $\mathrm{SH}$ helped to design the experiment and edited the manuscript. $\mathrm{AC}$ and $\mathrm{VG}$ helped designed the experiment and help to carry it out and edit the manuscript. VS and BT helped to design the experiment and edit the manuscript. PL helped to design the experiment and carry it out, analyse the data and edited the manuscript. All authors contributed to the article and approved the submitted version.

\section{FUNDING}

LY was funded by an NMRC Research Training Fellowship (NMRC/FLWSHP/043/2017) for this study.

\section{SUPPLEMENTARY MATERIAL}

The Supplementary Material for this article can be found online at: https://www.frontiersin.org/articles/10.3389/fdgth. 2021.824334/full\#supplementary-material

Supplementary Figure 1 | Rat 4 which did not show a reduction in ASL lesion volume with $\mathrm{CO}_{2}$ administration. The MRI sequences show a large infarct with involvement of the hypothalamic region (yellow arrow) that resulted in hyperthermia of the animal throughout the experiment.
10. Schmidt-Kastner R, Grosse Ophoff B, Hossmann K-A. Delayed recovery of $\mathrm{CO} 2$ reactivity after one hour's complete ischaemia of cat brain. J Neurol. (1986) 233:367-9. doi: 10.1007/BF00313924

11. Schmitz B. Bottiger BW, Hossmann K-A. Functional activation of cerebral blood flow after cardiac arrest in rat. J Cereb Blood Flow Metab. (1997) 17:1202-9. doi: 10.1097/00004647-199711000-00009

12. Nemoto EM, Snyder JV, Carroll RG, Morita H. Global ischemia in dogs: cerebrovascular CO2 reactivity and autoregulation. Stroke. (1975) 6:42531. doi: 10.1161/01.STR.6.4.425

13. Christopherson TJ, Milde JH, Michenfelder JD. Cerebral vascular autoregulation and $\mathrm{CO} 2$ reactivity following onset of the delayed postischemic hypoperfusion state in dogs. J Cereb Blood Flow Metab. (1993) 13:260-8. doi: 10.1038/jcbfm.1993.32

14. Fan YY, Shen Z, He P, Jiang L, Hou WW, Shen Y, et al. novel neuroprotective strategy for ischemic stroke: transient mild acidosis treatment by $\mathrm{CO} 2$ inhalation at reperfusion. J Cereb Blood Flow Metab. (2014) (2):27583. doi: $10.1038 /$ jcbfm. 2013.193

15. Olah L, Franke C, Schwindt W, Hoehn M. CO(2) reactivity measured by perfusion MRI during transient focal cerebral ischemia in rats. Stroke. (2000) (9):2236-44. doi: 10.1161/01.STR.31.9.2236

16. Thompson SW. Reactivity of cerebral blood flow to CO 2 in patients with transient cerebral ischemic attacks. Stroke. (1971) 2:273-8. doi: 10.1161/01.STR.2.3.273

17. Simon RP, Niro M, Gwinn R. Brain acidosis induced by hypercarbic ventilation attenuates focal ischemic injury. J Pharmacol Exp Ther. (1993) 267:1428-31.

18. Arnberg F, Lundberg J, Söderman M, Damberg P, Holmin S. Imageguided method in the rat for inducing cortical or striatal infarction and for controlling cerebral blood flow under MRI. Stroke. (2012) 43:243743. doi: 10.1161/STROKEAHA.112.655126

19. Little P, Kvist O, Grankvist R, Jonsson S, Damberg P, Soderman M, et al. Preserved Collateral Blood Flow in the Endovascular M2CAO Model Allows for Clinically Relevant Profiling of Injury Progression in 
Acute Ischemic Stroke. PLoS ONE. (2017) 12: e0169541. doi: 10.1371/ journal.pone.0169541

20. Mozaffarian D, Benjamin EJ, Go AS, Arnett DK, Blaha MJ, Cushman $\mathrm{M}$, et al. American Heart Association Statistics Committee and Stroke Statistics Subcommittee. Heart disease and stroke statistics--2015 update a report from the American Heart Association. Circulation. (2015) 131:e29322. doi: 10.1161/CIR.0000000000000152

21. Murray CJ, Vos T, Lozano R, et al. Disability-adjusted life years (DALYs) 1 diseases and injuries in 21 regions, 1990-2010: a systematic analysis for the Global Burden of Disease Study 2010. Lancet. (2012) 380:2197223. doi: 10.1016/S0140-6736(12)61689-4

22. Fisher M. The ischemic penumbra: identification, evolution and treat-ment concepts. Cerebrovasc Dis. (2004) 17:1-6. doi: 10.1159/ 000074790

23. Olivot JM, Mlynash M, Inoue M, Marks MP, Wheeler HM, Kemp $\mathrm{S}$, et al. Hypoperfusion intensity ratio predicts infarct progression and functional outcome in the DEFUSE 2 Cohort. Stroke. (2014) 45:101823. doi: 10.1161/STROKEAHA.113.003857

24. Wheeler HM, Mlynash M, Inoue M, Tipirnini A, Liggins J, Bammer R, et al. The growth rate of early DWI lesions is highly variable and associated with penumbral salvage and clinical outcomes following endovascular reperfusion. Int J Stroke. (2015) 10:723 \pm 9 . doi: 10.1111/ijs.12436

25. Scalzo F, Nour M, Liebeskind DS. Data science of stroke imaging and enlightenment of the penumbra. Front Neurol. (2015) 6:8. doi: 10.3389/fneur.2015.00008

26. Kety SS, Schmidt CF. The effects of altered arterial tensions of carbon dioxide and oxygen on cerebral blood flow and cerebral oxygen consumption of normal young men. J Clin Invest. (1948) 4:484-92. doi: 10.1172/ JCI101995

27. Fenton RA, Rubio R, Berne RM. Adenosine and the acidbase state of vascular smooth muscle. J Appl Physiol. (1981) 51:179-84. doi: 10.1152/jappl.1981.51.1.179

28. Karaki H, Weiss GB. Effect of transmembrane $\mathrm{pH}$ gradient changes on potassium-induced relaxation in vascular smooth muscle. Blood Vessels. (1981) 18:36-44. doi: 10.1159/000158336

29. Grubb RL, Raichle ME, Eichling JO, Ter-Pogossian MM. The effects of changes in $\mathrm{PaCO} 2$ on cerebral blood volume, blood flow, and vascular mean transit time. Stroke. (1974) 5:630-9. doi: 10.1161/01.STR.5.5.630

30. Ruta TS, Drummond JC, Cole DJ. The effect of acute hypocapnia on local cerebral blood flow during middle cerebral artery occlusion in isofluorane anesthetized rats. Anesthesiology.

(1993) 78:134-40. doi: 10.1097/00000542-199301000-00019

31. Koizumi G, Yoshida J, Nakazawa Y, Ooneda T. Experimental studies of ischemic brain edema: I: a new experimental model of cerebral embolism in rats in which recirculation can be introduced in the ischemic area. Jpn J Stroke. (1986) 8:8. doi: 10.3995/jstroke.8.1

32. Longa EZ, Weinstein PR, Carlson S, Cummins R. Reversible middle cerebral artery occlusion without craniectomy in rats. Stroke. (1989) 20:8491. doi: 10.1161/01.STR.20.1.84

33. Carmichael ST. Rodent models of focal stroke: size, mechanism, and purpose. NeuroRx. (2005) 2:396-409. doi: 10.1602/neurorx.2.3.396

34. Kanemitsu H, Nakagomi T, Tamura A, Tsuchiya T, Kono G, Sano K. Differences in the extent of primary ischemic damage between middle cerebral artery coagulation and intraluminal occlusion models. J Cereb Blood Flow Metab. (2002) 22:1196士204. doi: 10.1097/01.wcb.0000037992.07114.95

35. Brown JO. The morphology of circulus arteriosus cerebri in rats. Anat Rec. (1966) 156:99-106. doi: 10.1002/ar.1091560112

36. Coyle P. Arterial patterns of the rat rhinencephalon and related structures. Exp Neurol. (1975) 49:671-90. doi: 10.1016/0014-4886(75)90051-5

37. McAuley MA. Rodent models of focal ischemia. Cerebrovasc Brain Metab Rev. (1995) 7:153-80.

Conflict of Interest: The authors declare that the research was conducted in the absence of any commercial or financial relationships that could be construed as a potential conflict of interest.

Publisher's Note: All claims expressed in this article are solely those of the authors and do not necessarily represent those of their affiliated organizations, or those of the publisher, the editors and the reviewers. Any product that may be evaluated in this article, or claim that may be made by its manufacturer, is not guaranteed or endorsed by the publisher.

Copyright (C) 2022 Yeo, Arnberg, Chireh, Sharma, Tan, Gontu, Little and Holmin. This is an open-access article distributed under the terms of the Creative Commons Attribution License (CC BY). The use, distribution or reproduction in other forums is permitted, provided the original author(s) and the copyright owner(s) are credited and that the original publication in this journal is cited, in accordance with accepted academic practice. No use, distribution or reproduction is permitted which does not comply with these terms. 